\title{
STABILITY OF UV/OZONE TREATMENT OF PLASTICS FOR CAPILLARY-DRIVEN MICROFLUIDICS
}

\author{
Tung-Yi Lin and Peter B. Lillehoj* \\ Michigan State University, East Lansing, Michigan, USA
}

\begin{abstract}
In this paper, we report on the long-term stability of UV/Ozone (UVO) treatment of plastics which is a simple method to generate hydrophilic surfaces for capillary-driven flows in microfluidic devices. Previous reports on the stability of UVO-treated plastics were limited to 4 weeks. Here, we present new findings on the longterm stability of UVO-treated plastics for up to 16 weeks and also demonstrate for the first time that the storage condition has a significant impact on the surface stability. In particular, we show that the hydrophobic recovery of some UVO-treated plastics can be inhibited by storing them in dehumidified or vacuum conditions, making them useful for applications requiring long-term storage.
\end{abstract}

\section{INTRODUCTION}

Microfluidics is a promising technology that has been applied to many biomedical applications including cell biology, pharmacology and medical diagnosis $[1,2]$. These applications generally require precise control of liquids in a timely manner which involves the use of bulky or costly equipment. Alternatively, accurate displacement of microfluidic flows can be achieved using actuated on-chip micropumps [3, 4]. While micropumps can provide precise, configurable flow rates, they increase the complexity and costs associated with microfluidic devices. The realization of microfluidic platforms that are simple, portable and inexpensive requires alternative means for fluid transport without externally-actuated components. Towards this end, passive pumping is a promising approach that doesn't require any moving components and can greatly simplify device fabrication and operation.

Among passive pumping methods, one of the most common is capillary pumping which employs surface tension forces to 'pull' liquids through capillaries and microchannels. This approach also offers simplicity in controlling the flow rate which mainly depends on the liquid viscosity and geometry and surface properties of the channels $[5,6]$. In particular, the most important parameter for generating capillary flow is the surface hydrophilicity. Therefore, by controlling the hydrophilicity of the channel surfaces, it is possible to design microfluidic systems capable of precise, autonomous flow rates.

Previous reports have demonstrated capillary flows in silica and glass devices [7-9], however, these materials require complicated micromachining methods and are difficult to dispose. Capillary flows in polydimethylsiloxane (PDMS) microchannels have also been demonstrated using chemical surface treatments [10], however, PDMS is gas permeable and incompatible with some chemicals. Thermoplastic polymers have been extensively investigated over the past decade as a suitable material for microfluidic devices due to their low cost, disposability, optical transparency and biocompatibility [11]. One major drawback of plastics is that they are mildly hydrophilic in their native form, limiting their use for capillary flows. The surface of plastics can be made more hydrophilic by various surface treatments. For example, hydrophilic chemicals have been grafted onto plastic surfaces [12]. However, these methods can involve long and complicated fabrication processes and/or require the use of expensive equipment. An alternative approach is to oxidize the surface via exposure to an oxygen $\left(\mathrm{O}_{2}\right)$ plasma [13]. However, the stability of oxidized surfaces is poor, especially for PDMS where the surface undergoes hydrophobic recovery within a few hours [14].
Recently, a UVO treatment was demonstrated for generating hydrophilic surfaces on cyclic olefin copolymer (COC) which remained stable for up to 4 weeks [15]. In addition to enhanced stability, surface oxidization can also achieve thermal bonding at low process temperatures by preventing bulking and deformation of microfluidic structures [16]. While UVO treatment is a promising approach to enhance the surface hydrophilicity of plastic, further work is needed to evaluate its stability for applications requiring long-term storage ( $>4$ weeks).

In this work, we studied the stability of UVO-treated plastics under different storage conditions for up to 16 weeks. We focused our efforts on poly(methyl methacrylate) (PMMA), polycarbonate (PC), and COC because they are the most attractive plastics for biomedical applications due to their excellent optical transparency, biocompatibility and manufacturability [17]. Surface characterization of UVO-treated and untreated plastic samples was performed using x-ray photoelectron spectroscopy (XPS) and contact angle measurements. PMMA microchannels were fabricated and tested to demonstrate the effectiveness of UVO treatment for generating capillary-driven flows.

\section{MATERIALS AND METHODS}

\section{Sample Preparation}

PC and PMMA were purchased from McMaster-Carr (Elmhurst, IL) and COC was purchased from Zeon Chemicals (Louisville, KY). For stability studies, COC and PMMA were cut into $1 \mathrm{~cm} \times 2 \mathrm{~cm}$ pieces using a $\mathrm{CO}_{2}$ laser cutter (Universal Laser Systems, Scottsdale, AZ) and PC was cut using a band saw. UVO treatment was performed using a UVO cleaner (Novascan Technologies, Ames, IA). For stability studies, samples were treated for $20 \mathrm{~min}, 40 \mathrm{~min}, 60 \mathrm{~min}$ or $80 \mathrm{~min}$ at room temperature and stored in air, a desiccator or vacuum-sealed bags (Weston vacuum sealer, Strongsville, $\mathrm{OH}$ ).

\section{Contact Angle Measurements}

Static contact angle measurements were carried out using a VCA-2000 video contact angle analysis system (AST Products Inc., Billerica, MA). $0.5 \mu \mathrm{L}$ droplets of distilled water were dispensed onto the samples using a pipette. Contact angle data was acquired at ambient conditions from three different samples at a minimum of three locations per sample. The data is plotted as the mean value for eight separate measurements with outliers removed. Fresh samples were used for each set of measurements.

\section{XPS Analysis}

XPS spectra were obtained using a Perkin Elmer Phi 5400 ESCA system (Physical Electronics, Chanhassen, MN) at pressures between $10^{-9}$ and $10^{-8}$ torr, pass energy of $29.35 \mathrm{eV}$, and a $45^{\circ}$ takeoff angle. Elemental composition were calculated from the relative intensities of the $\mathrm{C} 1 \mathrm{~s}$ and $\mathrm{O} 1 \mathrm{~s}$ peak areas obtained from the survey spectral after subtraction of a linear background.

\section{Microchannel Fabrication}

Microfluidic devices were fabricated by cutting microfluidic features in PMMA using a laser cutter, applying UVO treatment and bonding the pieces together using double-sided adhesive film (Adhesive Research, Glen Rock, PA). The devices consist of three 
layers, as shown in Fig. 1. The width, height and length of the channel are $150 \mu \mathrm{m}, 1.87 \mathrm{~mm}$ and $30 \mathrm{~mm}$, respectively, and the diameter of the inlet and outlet is $2 \mathrm{~mm}$.

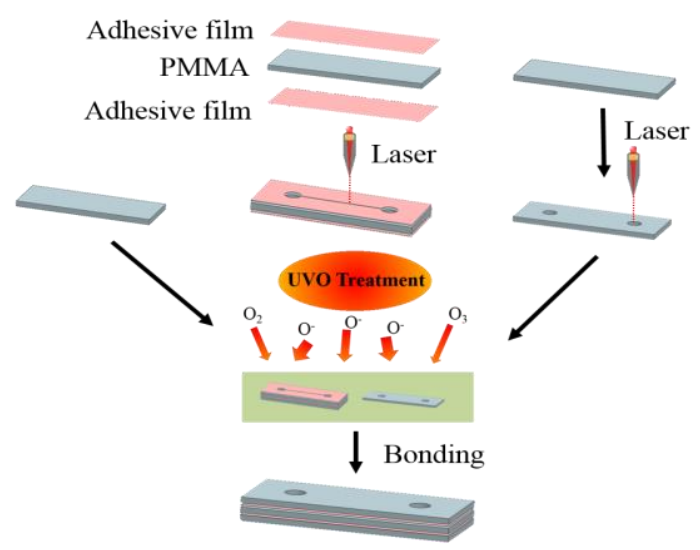

Figure 1: Fabrication process flow for PMMA microchannels.

\section{RESULTS AND DISCUSSION}

Influence of UVO Treatment on Surface Hydrophilicity

Contact angle measurements were performed on PMMA, PC and COC samples to investigate the influence of UVO treatment time on surface hydrophilicity. The data is plotted as the relative change in the contact angle (CA) following UVO treatment:

$$
\text { Relative change in } C A=\frac{\text { Treated } C A-\text { Non treated } C A}{\text { Non treated } C A} \times 100 \% \text { (1) }
$$

As shown in Fig. 2, UVO-treated samples exhibit significant reductions in contact angle compared with untreated samples for all three plastics. The contact angle of COC and PC drop significantly by $60.6 \% \pm 2.5 \%$ and $70.1 \% \pm 3.2 \%$, respectively, after $20 \mathrm{~min}$ of treatment, whereas PMMA exhibits a less significant reduction $(31.0 \% \pm 2.9 \%)$. All three plastics experience further reductions in contact angle with longer treatment times. Among the three plastics, PC exhibits the largest reduction in contact angle compared with PMMA and COC: after 80 min of UVO treatment, the contact angle of PC is reduced by $87.8 \% \pm 0.8 \%$. In contrast, the contact angle of COC does not significantly change with longer treatment times. Our results for COC match well with those reported by Bhattacharyya et al [18], who also showed a negligible change in contact angle with treatment times $>20$ min. For PMMA, a correlation between the treatment time and surface hydrophilicity was observed where longer times resulted in higher relative changes in contact angle. These results are consistent with those reported by Tsao et al [19] who also showed that the contact angle of PMMA is proportional to UVO treatment time. Based on this approach, the surface hydrophilicity of plastics can be tuned by simply adjusting the UVO treatment time.

UVO-induced surface oxidation is facilitated by $185 \mathrm{~nm}$ and $254 \mathrm{~nm}$ ultraviolet light which results in the removal of organic contaminants and non-destructive atomic layer etching. In the presence of oxygen, $185 \mathrm{~nm}$ light generates ozone while $254 \mathrm{~nm}$ light simultaneously excites organic molecules on the surface. The $254 \mathrm{~nm}$ light also generates free radicals on the surface that react with oxygen, thereby generating more oxygen-containing species [19]. Therefore, the degree of surface oxidation can be determined by investigating the surface chemistry. XPS measurements were performed on untreated and UVO-treated plastic samples to examine

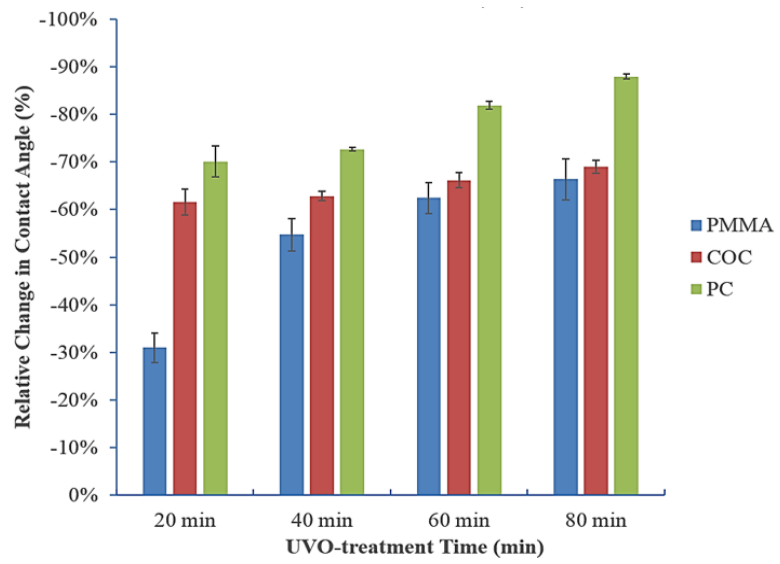

Figure 2: Relative change in contact angle of PMMA, COC and PC after UVO treatment. Error bars represent the mean \pm standard deviation (SD) of eight measurements.

the changes in surface chemistry resulting from the treatment process (Table 1). XPS measurements immediately following $80 \mathrm{~min}$ of UVO treatment are shown in Table 1 (a), which reveal that UVOtreated samples exhibit significantly higher oxygen/carbon $(\mathrm{O} / \mathrm{C})$ ratios compared with untreated samples. This is due the introduction of additional oxygen species on the polymer surface, indicative of the formation of the hydrophilic oxide layer. This data supports our contact angle measurements (Fig. 2) which show that UVO treatment enhances surface hydrophilicity. XPS measurements of UVO-treated samples after 16 weeks under different storage conditions are shown in Table 1(b). The O/C ratios for all stored samples are lower than those of freshly UVO-treated samples. Samples stored in dehumidified conditions exhibit the largest $\mathrm{O} / \mathrm{C}$ ratios compared with those stored in vacuum or air for all plastics. Interestingly, the $\mathrm{O} / \mathrm{C}$ ratios for plastic samples stored in vacuum are only slightly higher than those for samples stored in air. These results suggest that under proper storage conditions, UVO-treated plastics can retain a robust oxide layer and maintain surface hydrophilicity.

Table 1: XPS analysis of the O/C ratio on the surface of UVOtreated and untreated plastics immediately following treatment (a) and after 16 weeks under different storage conditions (b).

\begin{tabular}{cccc}
\hline (a) & PMMA & COC & PC \\
\hline Non-treated & 0.32 & 0.20 & 0.19 \\
Treated & 0.48 & 0.72 & 0.44 \\
\hline (b) & PMMA & COC & PC \\
\hline Air & 0.27 & 0.31 & 0.34 \\
Dehumidified & 0.41 & 0.38 & 0.38 \\
Vacuum & 0.34 & 0.34 & 0.36 \\
\hline
\end{tabular}

\section{Stability of UVO-Treated Surfaces}

In addition to analyzing the surface chemistry, we investigated the stability of UVO-treated samples by performing static contact angle measurements for 16 weeks. Measurements were performed on samples that were treated for $80 \mathrm{~min}$ and stored under different conditions (vacuum, dehumidified and air). As shown in Fig. 3, the contact angle of all three plastics gradually increase over time as a result of hydrophobic recovery. Among the three storage conditions, samples stored in air exhibited the most substantial hydrophobic 

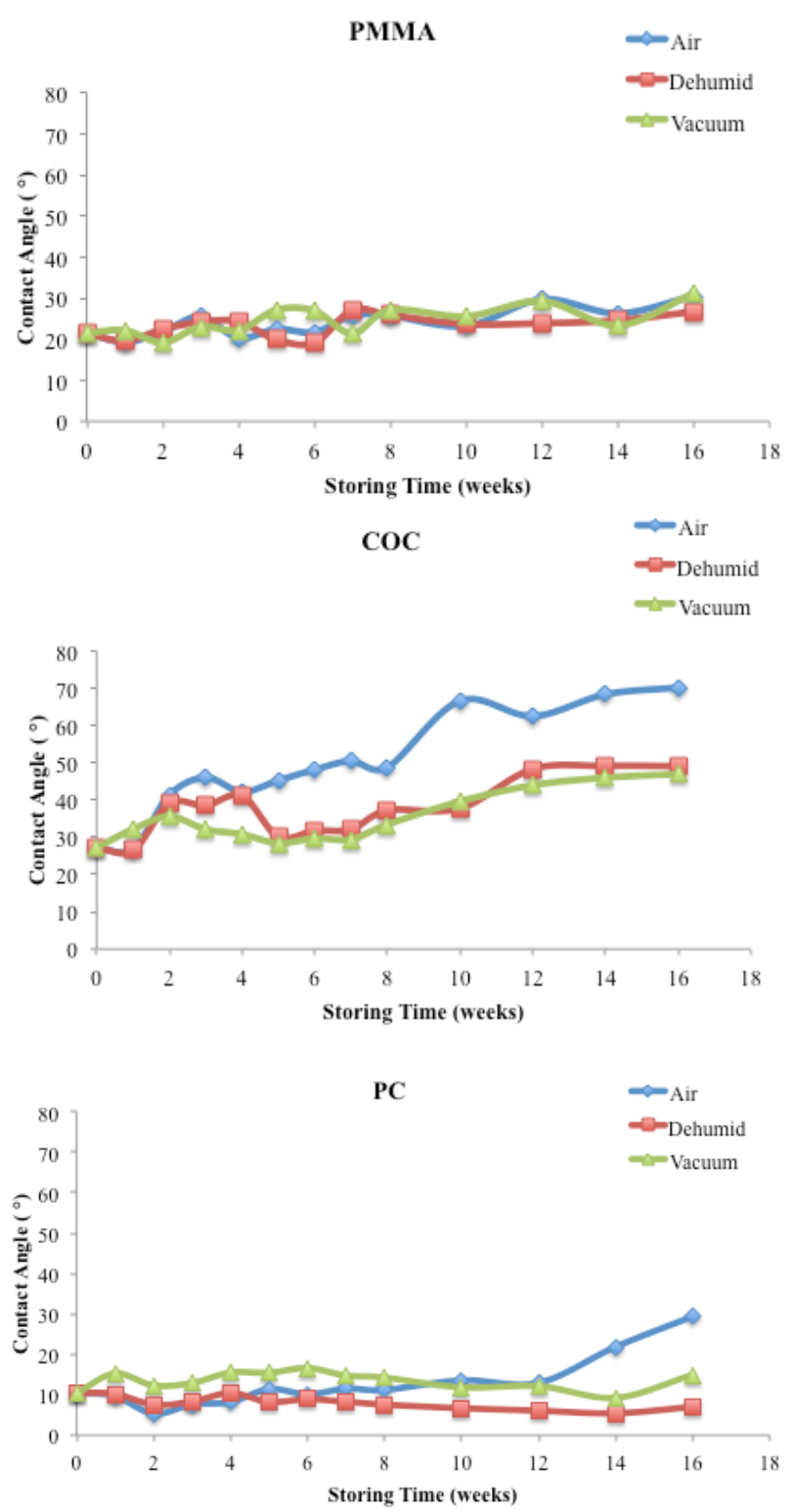

Figure 3: Long-term contact angle measurements of UVO-treated PMMA, COC and PC under various storage conditions. Each data point represents the mean of eight measurements.

recovery. In particular, the contact angle of COC increased significantly after only 3 weeks whereas PC and PMMA were stable for up to 12 weeks and 16 week, respectively. Interestingly, the storage condition had a negligible impact on the stability of PMMA, which remained stable for the entire duration of this study (16 weeks). In contrast, the degree of hydrophobic recovery for COC and PC was greatly influenced by the storage condition. Specifically, the contact angle of PC stored in air increased by $\sim 180 \%$ compared with dehumidified samples whereas vacuumstored samples increased by only $\sim 40 \%$. For COC, the contact angle of samples stored in air and vacuum increased by $\sim 160 \%$ and $\sim 75 \%$, respectively compared with dehumidified samples. These results are consistent with the XPS measurements presented in Table 1 which showed that the sample stored in air exhibited the lowest $\mathrm{O} / \mathrm{C}$ ratio while the dehumidified sample exhibited the highest $\mathrm{O} / \mathrm{C}$ ratio. Based on these collective results, we show that storing UVO-treated
PC and COC samples in dehumidified and vacuum conditions can substantially reduce their hydrophobic recovery by preserving surface oxidation and minimizing surface contamination, thus improving overall surface stability.

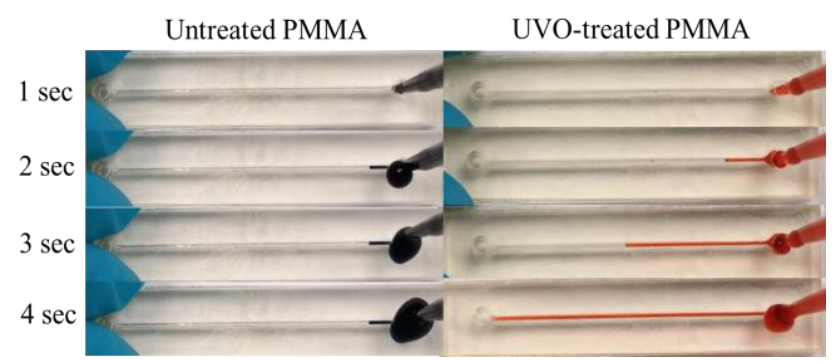

Figure 4: Capillary flow in untreated (left) and 80 min UVO-treated (right) PMMA microchannels. Colored liquid is dispensed at the inlet of the chips using a pipette.

\section{Effectiveness of UVO Treatment for Capillary Flow}

To demonstrate the effectiveness of UVO-treated microchannels for capillary-driven flow, we performed flow experiments using PMMA microchannels with varying UVO treatment times. Experiments were also carried out using untreated PMMA microchannels for comparison. $40 \mu \mathrm{L}$ of colored liquid was manually dispensed into the inlet of the microchannels using a pipette. As shown in Fig. 4, liquids quickly filled the 80 min-UVOtreated microchannel in $<4 \mathrm{sec}$ while negligible flow was observed in the untreated microchannel. We also measured the volume flow rate for microchannels with various UVO treatment times (Fig. 5). From this plot, we observe that there is a clear correlation between the treatment time and the flow rate due to the enhanced surface hydrophilicity generated from longer UVO exposure. This method can be applied to more complex microfluidic networks to develop capillary-driven systems that offer enhanced automation for more simplified operation. These results also suggest that UVO-treated microchannels can remain effective in pumping liquids via capillary flow after prolonged periods of storage when properly stored in dehumidified or vacuum conditions.

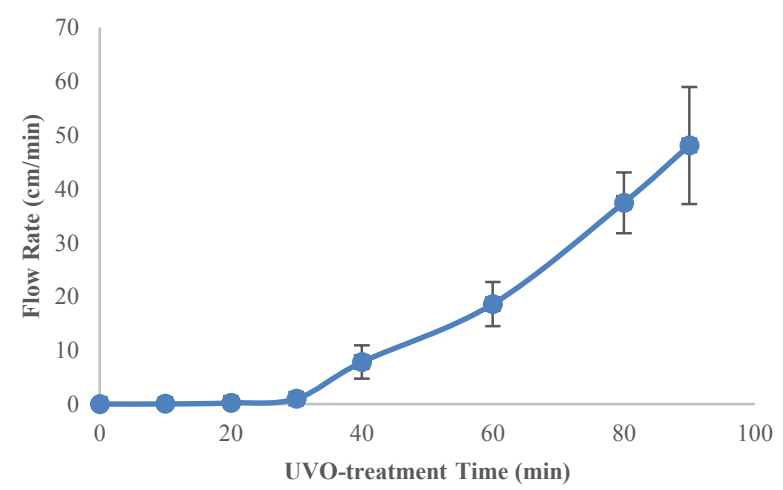

Figure 5: Volume flow rate as a function of UVO treatment time for $P M M A$ microchannels. Each data point represents the mean $\pm S D$ of three measurements.

\section{CONCLUSION}

UVO treatment is a simple method for generating hydrophilic surfaces on plastic, making it particularly useful for capillary flowbased microfluidic applications. In this work, we showed that UVO treatment time has a significant impact on the surface hydrophilicity of PC, COC and PMMA where longer times resulted in greater 
hydrophilicity. XPS measurements revealed that the enhanced hydrophilicity in UVO-treated plastics was due to a higher oxygen content in the polymer surface. Long-term contact angle measurements showed that UVO-treated samples exhibit significant hydrophobic recovery when stored in air, especially for PC and COC, whereas surface stability could be maintained for up to 16 weeks when stored in dehumidified or vacuum conditions. Lastly, we demonstrated the effectiveness of UVO treatment on PMMA microchannels for generating capillary-driven flows. With further work, this approach can facilitate the development of low cost plastic microfluidic devices for important biomedical applications.

\section{ACKNOWLEDGEMENT}

This work was funded by the National Institutes of Health (R01 AI113257). We thank Kathryn Severin for her assistance with contact angle measurements.

\section{REFERENCES}

[1] E. K. Sackmann, A. L. Dulton and D. J. Beebe, "The present and future role of microfluidics in biomedical research", Nature, 507, 181 (2014).

[2] G. M. Whitesides, "The origins and the future of microfluidics", Nature, 442, 368 (2006).

[3] P. Woias, "Micropumps-past, progress and future prospects", Sens. Actuator. B, 105, 28 (2005).

[4] D. J. Laser and J. G. Santiago, "A review of micropumps", Journal of Micromechanics and Microengineering, 14, 6 (2004).

[5] D. Juncker, H. Schmid, U. Drechsler, H. Wolf, M. Wolf, B. Michel, N. de Rooij and E. Delamarche, "Autonomous Microfluidic Capillary System”, Anal Chem, 74, 6139 (2002).

[6] M. Zimmermann, H. Schmid, P. Hunziker and E. Delamarche, "Capillary pumps for autonomous capillary systems", Lab Chip, 7, 119 (2007).

[7] B. Zhao, J. S. Moore and D. J. Beebe, "Surface-directed liquid flow inside microchannels", Science, 291, 1023 (2001).

[8] P. Lam, K. J. Wynne and G. E. Wnek, "Surface-TensionConfined Microfluidics", Langmuir, 18, 948 (2002).

[9] K. B. Lee and L. Lin, "Surface micromachined glass and polysilicon microchannels using MUMPs for BioMEMS applications", Sensor Actuat A-Phys, 111, 44 (2004).

[10] E. Delamarche, A. Bernard, H. Schmid, B. Michel and H. Biebuyck, "Patterned delivery of immunoglobulins to surfaces using microfluidic networks", Science, 276, 779 (1997).

[11] S. A. Soper, S. M. Ford, R.L. McCarley, K. Kelly and M. Murphy, "Polymeric microelectro-mechanical systems", Anal Chem, 72, 642 (2000).

[12] C. Jonsson, M. Aronsson, G. Rundstrom, C Pettersson, I. Mendel-Hartvig, J. Bakker et al, "Silane-dextran chemistry on lateral flow polymer chips for immunoassays", Lab Chip, 8, 1191 (2008).

[13] I. Beaulieu, M. Geissler and J. Mauzeroll, "Oxygen plasma treatment of polystyrene and zeonor: substrates for adhesion of patterned cells", Langmuir, 24, 12 (2009).

[14] D. Bodas and C. Khan-Malek, "Hydrophilization and hydrophobic recovery of PDMS by oxygen plasma and chemical treatment - An SEM investigation", Sens and Actuators B, 123, 368 (2007).

[15] P. M. Midwoud, Arnout Janse, M. T. Merema, G. M. Groothuis, E. Verpoorte, "Comparison of Biocompatibility and Adsorption Properties of Different Plastics for Advanced Microfluidic Cell and Tissue Culture Models", Anal Chem, 84, 3938 (2012).

[16] P. Kettner, R. L. Pelzer, T. Glinsner and S. Farrens, J. Phys.: Conf. Series, 34, (2006), pp. 65-71.

[17] A. Piruska, I. Mikcevic, S. H. Lee, C. Ahn, W. R. Heineman, P. A. Limbach and C. J. Seliskar, "The Autofluorescence of Plastic Materials and Chips Measured under Laser Irradiation", Lab Chip, 5, 1348 (2005).

[18] A. Bhattacharyya and C.M. Klapperich, "Mechanical and chemical analysis of plasma and ultraviolet-ozone surface treatments for thermal bonding of polymeric microfluidic devices", Lab Chip, 7, 876 (2007).

[19] C. W. Tsao, L. Hromada, J. Liu, P. Kumar and D. L. DeVoe, "Low temperature bonding of PMMA and COC microfluidic substrates using UV/ozone surface treatment”, Lab chip, 7, 499 (2007).

\section{CONTACT}

*P.B. Lillehoj, Tel: +1-517-432-2975; 1illehoj@egr.msu.edu 